\title{
Simultaneous reconstruction of absorbed optical energy density and speed of sound distributions in photoacoustic computed tomography
}

Chao Huang, Kun Wang, Robert W. Schoonover, Lihong V. Wang, Mark A. Anastasio

Chao Huang, Kun Wang, Robert W. Schoonover, Lihong V. Wang, Mark A. Anastasio, "Simultaneous reconstruction of absorbed optical energy density and speed of sound distributions in photoacoustic computed tomography," Proc. SPIE 8943, Photons Plus Ultrasound: Imaging and Sensing 2014, 894360 (3 March 2014); doi: 10.1117/12.2041825

SPIE. Event: SPIE BiOS, 2014, San Francisco, California, United States 


\title{
Simultaneous Reconstruction of Absorbed Optical Energy Density and Speed of Sound Distributions in Photoacoustic Computed Tomography
}

\author{
Chao Huang, Kun Wang, Robert W. Schoonover, Lihong V. Wang, and Mark A. Anastasio \\ Department of Biomedical Engineering, Washington University in St. Louis, \\ St. Louis, MO 63130
}

\begin{abstract}
An important and interesting question in photoacoustic computed tomography (PACT) is whether the absorbed optical energy density distribution, $A(\mathbf{r})$, and the speed of sound distribution, $c(\mathbf{r})$, can both be accurately determined from the measured photoacoustic data alone. However, in many cases $c(\mathbf{r})$ is unknown or cannot be accurately estimated. Therefore, it would be practically beneficial if $A(\mathbf{r})$ and $c(\mathbf{r})$ can be jointly reconstructed from the measurements. In this work, we propose a reconstruction approach to the joint reconstruction of both properties in PACT.
\end{abstract}

Keywords: Photoacoustic tomography, optoacoustic tomography, thermoacoustic tomography, iterative image reconstruction

\section{INTRODUCTION}

Conventional image reconstruction algorithms in photoacoustic computed tomography (PACT) are often based on idealized imaging models that assume an acoustically homogeneous medium. However, in applications such as transcranial PACT of primates, the photoacoustic (PA) wavefields can be strongly aberrated and attenuated by the skull. ${ }^{1-3}$ In such cases, if the acoustic heterogeneity is not accounted for in the reconstruction algorithm, the reconstructed images can contain significant distortions and artifacts. To compensate for the effects of acoustic heterogeneities, several image reconstruction methods have been proposed ${ }^{4-10}$ It has been demonstrated that, by incorporating the speed of sound (SOS) distribution, $c(\mathbf{r})$, into the imaging model, these methods can improve PACT image quality. However, in many cases of practical interest, $c(\mathbf{r})$ is unknown or cannot be accurately estimated.

This brings up an important question in PACT: can the absorbed optical energy density distribution, $A(\mathbf{r})$, and the speed of sound distribution, $c(\mathbf{r})$, both be accurately determined from the measured PA data alone? Recent work on the theory of joint reconstructions (JR) in PACT demonstrate only that $A(\mathbf{r})$ and $c(\mathbf{r})$ can be accurately determined from the measured data under certain restricted conditions, but the theoretical answer to general cases is still unknown. ${ }^{8,11}$ A few works have also reported the development of algorithms for JR of $A(\mathbf{r})$ and $c(\mathbf{r}) \cdot{ }^{12-14}$ In Ref. [12], an iterative reconstruction method was proposed to simultaneously estimate both $A(\mathbf{r})$ and $c(\mathbf{r})$. The method utilized a priori information regarding the geometry of the SOS distribution. In Refs. [13],[14], Jiang et al developed a JR method that was based on the Helmholtz equation, which was solved by the finite element method (FEM). While this method is grounded in a accurate model of the imaging physics, it suffers from an intensive computational burden.

In this study, we develop and investigate an optimization-based reconstruction approach to the JR of $A(\mathbf{r})$ and $c(\mathbf{r})$ that is based on the time-domain wave equation. The reconstruction method utilizes an alternating optimization scheme, where $A(\mathbf{r})$ is reconstructed by use of a previously-developed full-wave iterative method, ${ }^{10}$ while $c(\mathbf{r})$ is reconstructed by use of a nonlinear optimization algorithm based on the Fréchet derivative of an objective function with respect to $c(\mathbf{r}) \cdot{ }^{15,16}$ The developed JR method is investigated by use of computer simulation studies.

(Send correspondence to Mark A. Anastasio)

Mark A. Anastasio: E-mail: anastasio@seas.wustl.edu, Telephone: 13149357208

Photons Plus Ultrasound: Imaging and Sensing 2014, edited by Alexander A. Oraevsky, Lihong V. Wang, Proc. of SPIE Vol. 8943, 894360 - C 2014 SPIE · CCC code: 1605-7422/14/\$18 - doi: 10.1117/12.2041825 


\section{BACKGROUND}

In a heterogeneous lossless fluid medium where the variation of mass density can be neglected, the propagation of the thermoacoustically-induced pressure wavefield $p(\mathbf{r}, t)$ can be modeled by the following wave equation ${ }^{17,18}$

$$
\nabla^{2} p(\mathbf{r}, t)-\frac{1}{c(\mathbf{r})^{2}} \frac{\partial^{2} p(\mathbf{r}, t)}{\partial t^{2}}=0
$$

subject to initial conditions

$$
p(\mathbf{r}, 0)=\Gamma(\mathbf{r}) A(\mathbf{r}),\left.\quad \frac{\partial p(\mathbf{r}, t)}{\partial t}\right|_{t=0}=0,
$$

where $\Gamma(\mathbf{r})$ is the Grueneisen parameter that is assumed to be known throughout this paper.

Based on the wave equation, the inverse scattering problem of reconstruction of $c(\mathbf{r})$ for a known $A(\mathbf{r})$ can be formulated as a nonlinear optimization problem that estimates $c(\mathbf{r})$ by minimizing the objective functional:

$$
\mathcal{E}[c(\mathbf{r})]=\sum_{m=1}^{M} \int_{0}^{T} \mathrm{~d} t\left[p\left(\mathbf{r}^{m}, t\right)-\tilde{p}\left(\mathbf{r}^{m}, t\right)\right]^{2},
$$

subject to the constraint given by (1). Here, $\tilde{p}\left(\mathbf{r}^{m}, t\right)$ denotes the measured PA data from the $m$-th transducer at location $\mathbf{r}^{m}(m=1, \cdots, M)$ and $T$ denotes the maximum time at which the PA data were recorded. Note that, for simplicity, we neglect the acousto-electrical impulse response (EIR) of the ultrasonic transducers and assume each transducer is point-like in this study.

Gradient-based iterative algorithms can be utilized to minimize the nonlinear functional (3). Gradient-based methods require the functional gradient, or Fréchet derivative, of $\mathcal{E}$ with respect to $c(\mathbf{r})$, which can be calculated by use of the adjoint method. ${ }^{15,16}$ In the adjoint method, the adjoint wave equation is defined as

$$
\nabla^{2} q(\mathbf{r}, t)-\frac{1}{c(\mathbf{r})^{2}} \frac{\partial^{2} q(\mathbf{r}, t)}{\partial t^{2}}=-s(\mathbf{r}, t)
$$

subject to terminal conditions

$$
q(\mathbf{r}, T)=0,\left.\quad \frac{\partial q(\mathbf{r}, t)}{\partial t}\right|_{t=T}=0,
$$

where the source term is defined as

$$
s(\mathbf{r}, t)=\sum_{m=1}^{M}\left[p\left(\mathbf{r}^{m}, t\right)-\tilde{p}\left(\mathbf{r}^{m}, t\right)\right] \delta\left(\mathbf{r}-\mathbf{r}^{m}\right) .
$$

Upon solving (1) and (4), the Fréchet derivative of $\mathcal{E}$ with respect to $c(\mathbf{r})$ can be determined as, ${ }^{15,16}$

$$
\nabla_{c} \mathcal{E}=-\frac{4}{c(\mathbf{r})^{3}} \int_{0}^{T} \mathrm{~d} t \frac{\partial p(\mathbf{r}, t)}{\partial t} \frac{\partial q(\mathbf{r}, t)}{\partial t} .
$$

Once the Fréchet derivative is obtained, it can be utilized by any gradient method as the search direction to reconstruct $c(\mathbf{r})$.

\section{SIMULTANEOUS RECONSTRUCTION}

The Fréchet derivative described above permits us to formulate the JR of $A(\mathbf{r})$ and $c(\mathbf{r})$ as an alternating optimization problem that consists of two sub-problems: reconstruction of $A(\mathbf{r})$ given $c(\mathbf{r})$ and reconstruction of $c(\mathbf{r})$ given $A(\mathbf{r})$. To numerically solve the alternating optimization problem, a discrete imaging model of PACT needs to be established first, which is described below. 


\subsection{Discrete imaging model}

Let

$$
\mathbf{A} \equiv\left[A\left(\mathbf{r}_{1}\right), \cdots, A\left(\mathbf{r}_{N}\right)\right]^{\mathrm{T}}
$$

and

$$
\mathbf{c} \equiv\left[c\left(\mathbf{r}_{1}\right), \cdots, c\left(\mathbf{r}_{N}\right)\right]^{\mathrm{T}}
$$

be $N \times 1$ vectors representing the discretized $A(\mathbf{r})$ and $c(\mathbf{r})$ sampled at $N$ vertices on a Cartesian grid, respectively.

Likewise, we define

$$
\tilde{\mathbf{p}}_{l} \equiv\left[\tilde{p}\left(\mathbf{r}^{1}, l \Delta t\right), \cdots, \tilde{p}\left(\mathbf{r}^{M}, l \Delta t\right)\right]^{\mathrm{T}}
$$

as the measured PA data sampled at transducer locations $\mathbf{r}^{m}(m=1, \cdots, M)$ and at time $t=l \Delta t(l=$ $0, \cdots, L-1)$, Here, $\Delta t$ is the sampling time step, and $L$ is the total number of time steps.

The measured PA data corresponding to all transducer locations and temporal samples can then be defined as $L M \times 1$ vectors

$$
\tilde{\mathbf{p}} \equiv\left[\tilde{\mathbf{p}}_{0}, \cdots, \tilde{\mathbf{p}}_{L-1}\right]^{\mathrm{T}}
$$

By use of (8), (9), and (11), the discrete imaging model of PACT can be expressed as ${ }^{10}$

$$
\tilde{\mathbf{p}}=\mathbf{H}(\mathbf{c}) \mathbf{A},
$$

where $\mathbf{H}(\mathbf{c})$ is the $L M \times N$ system matrix, which depends on $\mathbf{c}$. The procedure to establish the explicit matrix representation of $\mathbf{H}(\mathbf{c})$ can be found in Ref. [10].

\subsection{Alternating optimization}

Based on the discrete imaging model (12), the inverse problem of reconstructing $\mathbf{A}$ for a given $\mathbf{c}$ can be formulated as the penalized least squares problem

$$
\hat{\mathbf{A}}=\underset{\mathbf{A} \geq 0}{\arg \min }\|\mathbf{H}(\mathbf{c}) \mathbf{A}-\tilde{\mathbf{p}}\|^{2}+\lambda_{A} \mathrm{R}(\mathbf{A}),
$$

where $\lambda_{A}$ is the regularization parameter, and $\mathrm{R}(\mathbf{A})$ is a regularization term. Depending on the choice of $\mathrm{R}(\mathbf{A})$, there exist different optimization algorithms to efficiently solve (13). ${ }^{19}$ In this study, a total variation (TV) penalty is adopted, and the fast iterative shrinkage/thresholding algorithm (FISTA) is utilized to solve (13). ${ }^{10,20}$

Similarly, reconstruction of $\mathbf{c}$ for a given $\mathbf{A}$ can be accomplished by solving the following optimization problem

$$
\hat{\mathbf{c}}=\underset{\mathbf{c}>0}{\arg \min }\|\mathbf{H}(\mathbf{c}) \mathbf{A}-\tilde{\mathbf{p}}\|^{2}+\lambda_{c} \mathrm{R}(\mathbf{c}) .
$$

The gradient of (14) can be obtained by discretizing (7), which can be utilized by gradient methods as search direction to reduce the objective function value in (14). In this study, the TV-penalty is also used to regularize the reconstruction of $\mathbf{c}$, and the limited-memory BFGS (L-BFGS) algorithm is employed to solve (14). ${ }^{19,21}$ Finally, A and $\mathbf{c}$ can be simultaneously reconsructed by alternately solving (13) and (14).

\section{NUMERICAL STUDIES AND RESULTS}

\subsection{Numerical studies}

A preliminary numerical investigation of the proposed JR method was conducted. Figure 1 shows the numerical phantoms of $\mathbf{A}$ and $\mathbf{c}$ employed in this study. By use of the $\mathrm{k}$-Wave toolbox ${ }^{22}$ simulated pressure data corresponding to those phantoms were computed at 120 transducer locations that were evenly distributed on a circle of radius $15 \mathrm{~mm}$. At each transducer location, a total of 1,000 temporal samples of the simulated pressure data were computed at time step $\Delta t=40 \mathrm{~ns}$. The simulated pressure data were then contaminated by $3 \%$ (with respect to maximum value of noiseless data) additive white Gaussian noise. To avoid inverse crime,the simulated pressure data were generated on a Cartesian grid of $512 \times 512$ pixels with a pitch of $0.1 \mathrm{~mm}$. Simultaneous reconstructions were conducted on a grid of $256 \times 256$ pixels with a pitch of $0.2 \mathrm{~mm}$. The regularization parameters $\lambda_{A}=0.001$ and $\lambda_{c}=0.001$ were empirically selected for use in the studies involving noiseless data. For the studies involving noisy data, $\lambda_{A}=0.003$ and $\lambda_{c}=0.01$ were employed. 


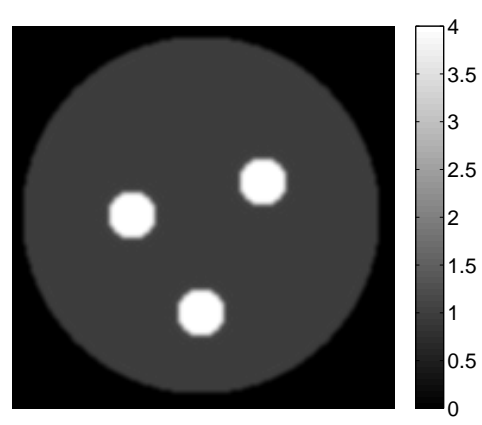

(a)

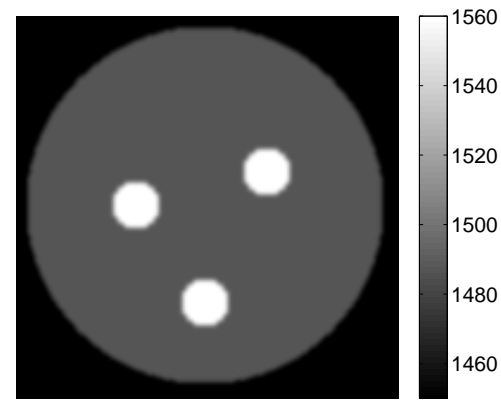

(b)

Figure 1.

\subsection{Numerical results}

The simultaneously reconstructed images corresponding to noiseless and noisy data are displayed in Figs. 2 and 3, respectively. In each figure, the results in the top row are the reconstructed $\mathbf{A}$ and its profile, while the bottom row shows the reconstructed $\mathbf{c}$ and its profile. The profiles are along the $\mathrm{X}$-axis indicated by the arrow in Fig. 2(a). The red lines and blue lines corresdpond to profiles through the phantom and reconstructed images, respectively. These results show that, in an idealized scenario where model errors can be neglected, it is possible to accurately reconstruct both $\mathbf{A}$ and $\mathbf{c}$ when appropriate regularization is employed. The noisy results also show that, by use of regularization, the proposed JR method is robust to measurement noise when no model errors exist.

\section{SUMMARY}

In this study, we investigated the JR problem in PACT by use of an alternating optimization method that is based on the wave equation. Computer simulation studies were conducted to assess the accuracy and the robustness of the proposed JR method. The numerical results showed that, in an idealized scenario where model errors can be neglected, it is possible to achieve accurate JR of $A(\mathbf{r})$ and $c(\mathbf{r})$ when appropriate regularization is employed. However, there always exist model errors in practice, such as inaccurate deconvolution of transducers' EIR. Therefore, the feasibility of accurate JR in practice remains an important topic for future study.

\section{ACKNOWLEDGMENTS}

This work was supported in part by NIH awards EB010049, EB01696301, and EB014617.

\section{REFERENCES}

1. Fry, F. J. and Barger, J. E., "Acoustical properties of the human skull," The Journal of the Acoustical Society of America 63(5), 1576-1590 (1978).

2. Jin, X., Li, C., and Wang, L. V., "Effects of acoustic heterogeneities on transcranial brain imaging with microwave-induced thermoacoustic tomography," Medical Physics 35(7), 3205-3214 (2008).

3. Huang, C., Nie, L., Schoonover, R. W., Guo, Z., Schirra, C. O., Anastasio, M. A., and Wang, L. V., "Aberration correction for transcranial photoacoustic tomography of primates employing adjunct image data," Journal of Biomedical Optics 17(6), 066016 (2012).

4. Xu, Y. and Wang, L., "Effects of acoustic heterogeneity in breast thermoacoustic tomography," Ultrasonics, Ferroelectrics and Frequency Control, IEEE Transactions on 50, 1134 -1146 (sept. 2003).

5. Modgil, D., Anastasio, M. A., and La Rivire, P. J., "Image reconstruction in photoacoustic tomography with variable speed of sound using a higher-order geometrical acoustics approximation," Journal of Biomedical Optics 15(2), 021308-021308-9 (2010). 


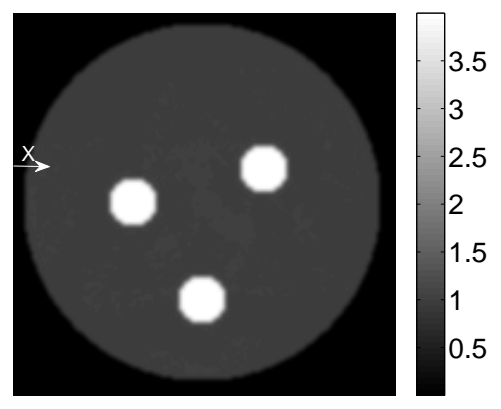

(a)

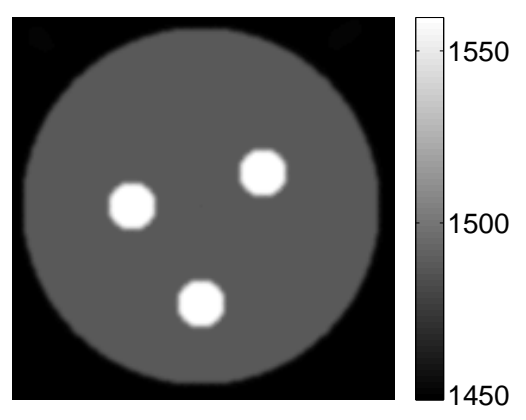

(c)

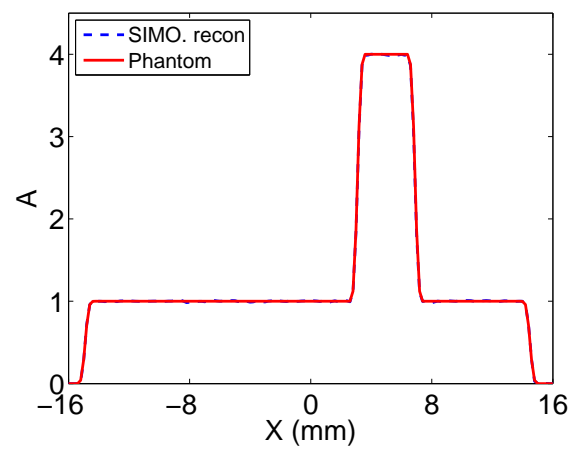

(b)

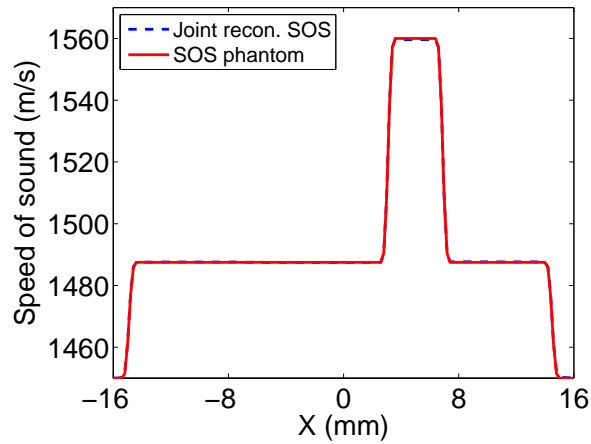

(d)

Figure 2. Reconstructed images corresponding to noiseless data. The top and bottom rows are the simultaneously reconstructed $\mathbf{A}$ and $\mathbf{c}$, respectively.

6. Jose, J., Willemink, R. G. H., Resink, S., Piras, D., van Hespen, J. C. G., Slump, C. H., Steenbergen, W., van Leeuwen, T. G., and Manohar, S., "Passive element enriched photoacoustic computed tomography (per pact) for simultaneous imaging of acoustic propagation properties and light absorption," Optics Express 19, 2093-2104 (Jan 2011).

7. Yuan, Z. and Jiang, H., "Three-dimensional finite-element-based photoacoustic tomography: Reconstruction algorithm and simulations," Medical Physics 34(2), 538-546 (2007).

8. Hristova, Y., Kuchment, P., and Nguyen, L., "Reconstruction and time reversal in thermoacoustic tomography in acoustically homogeneous and inhomogeneous media," Inverse Problems 24(5), 055006 (2008).

9. Stefanov, P. and Uhlmann, G., "Thermoacoustic tomography with variable sound speed," Inverse Problems 25(7), 075011 (2009).

10. Huang, C., Wang, K., Nie, L., Wang, L., and Anastasio, M., "Full-wave iterative image reconstruction in photoacoustic tomography with acoustically inhomogeneous media," Medical Imaging, IEEE Transactions on 32(6), 1097-1110 (2013).

11. Hickmann, K. S., Unique Determination of Acoustic Properties from Thermoacoustic Data, PhD thesis, Oregon State University (2010).

12. Zhang, J., Wang, K., Yang, Y., and Anastasio, M. A., "Simultaneous reconstruction of speed-of-sound and optical absorption properties in photoacoustic tomography via a time-domain iterative algorithm," Photons Plus Ultrasound: Imaging and Sensing 2008: The Ninth Conference on Biomedical Thermoacoustics, Optoacoustics, and Acousto-optics 6856, SPIE (2008).

13. Jiang, H., Yuan, Z., and Gu, X., "Spatially varying optical and acoustic property reconstruction using finiteelement-based photoacoustic tomography," The Journal of the Optical Society of America 23, 878-888 (Apr 2006). 


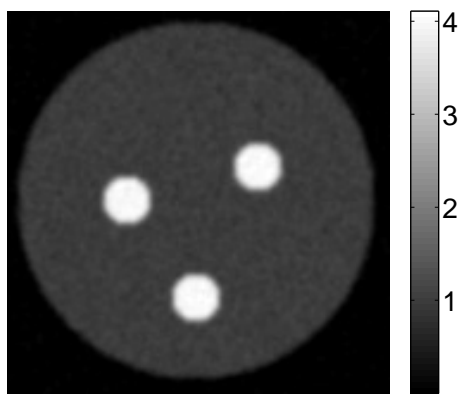

(a)

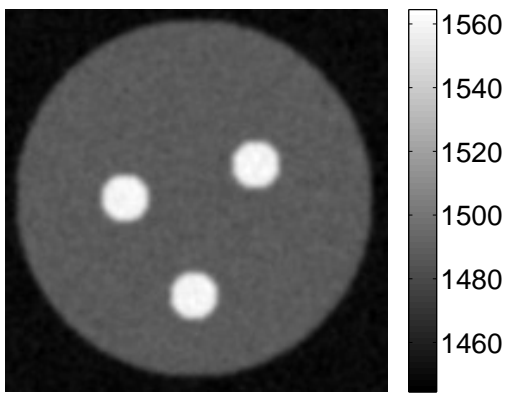

(c)

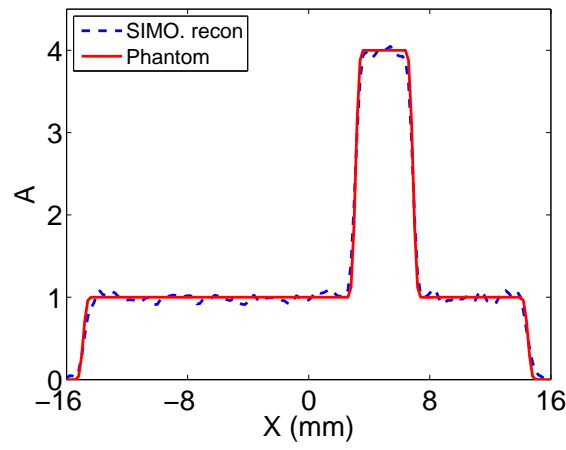

(b)

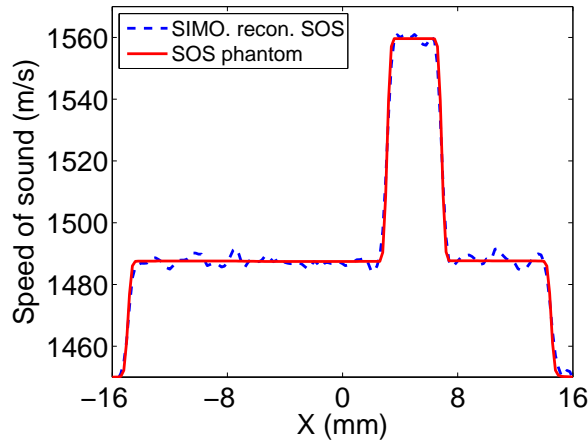

(d)

Figure 3. Reconstructed images corresponding to noisy data. The top and bottom rows are the simultaneously reconstructed $\mathbf{A}$ and $\mathbf{c}$, respectively.

14. Yuan, Z., Zhang, Q., and Jiang, H., "Simultaneous reconstruction of acoustic and optical properties of heterogeneous media by quantitative photoacoustic tomography," Optics Express 14, 6749-6754 (Jul 2006).

15. Bunks, C., Saleck, F., Zaleski, S., and Chavent, G., "Multiscale seismic waveform inversion," Geophysics 60(5), 1457-1473 (1995).

16. Norton, S. J., "Iterative inverse scattering algorithms: Methods of computing frechet derivatives," The Journal of the Acoustical Society of America 106(5), 2653-2660 (1999).

17. Morse, P. M., "Theoretical acoustics," in [Theoretical Acoustics], Princeton University Press (1987).

18. Wang, L. V., "Photoacoustic imaging and spectroscopy," in [Photoacoustic Imaging and Spectroscopy], CRC (2009).

19. Nocedal, J. and Wright, S. J., "Numerical optimization," in [Numerical Optimization], Springer (1999).

20. Beck, A. and Teboulle, M., "Fast gradient-based algorithms for constrained total variation image denoising and deblurring problems," Image Processing, IEEE Transactions on 18, 2419 -2434 (nov. 2009).

21. Dunlavy, D. M., Kolda, T. G., and Acar, E., "Poblano v1.0: A matlab toolbox for gradient-based optimization," Tech. Rep. SAND2010-1422, Sandia National Laboratories, Albuquerque, NM and Livermore, CA (Mar. 2010).

22. Treeby, B. and Cox, B., "k-wave: MATLAB toolbox for the simulation and reconstruction of photoacoustic wave fields," Journal of Biomedical Optics 15, 021314 (2010). 